\title{
Arbeitsgemeinschaft für Verhaltensmodifikation e.V. (AVM-D)
}

In der außerordentlichen Mitgliederversammlung am 17. 7. 93 wurden Änderungen des Ausbildungsmodells diskutiert und angenommen. Die Einzelheiten sind dem Protokoll der außerordentlichen Mitgliederversammlung zu entnehmen.

Die AK-Leiter- und Lehrtherapeuten-Konferenz am 24. 4. 93 befaßte sich mit den «Kriterien der Falldarstellung und Fallbegutachtung». Eine Standard-Falldarstellung wurde als nicht sinnvoll bewertet, Falldarstellungen sollten individuell und flexibel für den einzelnen Fall erfolgen. Nach ausführlicher Diskussion der Kriterien für eine Falldarstellung und Fallbegutachtung, in der sich als Konsens herausstellte, daß Gliederungen bestimmter Autoren nicht als Vorgabe dienen müssen - sie müssen ohnehin der Entwicklung angepaßt werden -, wurde eine Arbeitsgruppe gebildet, die Vorschläge für ein Gliederungsschema erarbeiten wird.

Die bisher nur von den Mitgliedern der AWANK geleistete Fallbegutachtung wird in Zukunft auch von den nicht zur AWANK gehörenden Lehrtherapeuten übernommen. Als zweites Thema stand der gemeindepsychologische Auftrag der AVM zur Diskussion. Die Notwendigkeit dieser Arbeit wird erkannt, sie sollte forciert werden, und neben einer Sammlung von Vorschlägen ging die Empfehlung an die Arbeitskreise, sich mit diesem Thema intensiv zu beschäftigen und Vorschläge für eventuell nötige Änderungen des Ausbildungsmodells zu machen (z. B. Wiederaufnahme von Projekten).

Die nächste AK-Leiter- und Lehrtherapeuten-Konferenz findet mit dem Thema «Selbsterfahrung» am 6.11.93 in Würzburg statt.

Unsere Literaturliste ist fast vergriffen, wird vom Verfasser Dieter Schmelzer auf den neuesten Stand gebracht und ist ab November 1993 wieder über unsere Geschäftsstelle zum Preis von DM 5,- (Schutzgebühr), bei Postversand DM 8,-, zu beziehen. Sie umfaßt 24 Seiten im Format DIN A4 (zuzüglich 3 Seiten Stichwortverzeichnis) und enthält aktuelle Literaturhinweise zu folgenden Schwerpunkten: Allgemeine Grundlagenliteratur zur VT und Klinischen Psychologie; Fachzeitschriften samt Bezugsadressen; Klinische Grundlagen und Basisfertigkeiten; Prozeßorientierte Techniken und Fertigkeiten; Störungsbilder; Standardtechniken; Sonderthemen (z. B. Umgang mit Mißerfolgen, Krisenintervention, Psychopharmaka, ethische Aspekte etc.). In München hat sich ein neuer Arbeitskreis gebildet (Kontaktadresse über die Geschäftsstelle). Geschäftsstelle AVM (D):

Lehrstuhl für Klinische Psychologie der Universität

Markusplatz 3

D-96047 Bamberg

Tel. (0951)863-1885 